\title{
Tight-binding model for hydrogen-silicon interactions
}

\author{
B. J. Min, Y. H. Lee, ${ }^{*}$ C. Z. Wang, C. T. Chan, and K. M. Ho \\ Microelectronics Research Center, Ames Laboratory, Iowa State University, Ames, Iowa 50011 \\ and Department of Physics and Astronomy, Ames Laboratory, Iowa State University, Ames, Iowa 50011
}

(Received 19 July 1991; revised manuscript received 1 November 1991)

\begin{abstract}
We have developed an empirical tight-binding model for use in molecular-dynamics simulations to study hydrogen-silicon systems. The hydrogen-silicon interaction is constructed to reproduce the electronic energy levels and vibration frequencies of silane $\left(\mathrm{SiH}_{4}\right)$. Further use of the model in the studies of disilane $\left(\mathrm{Si}_{2} \mathrm{H}_{6}\right)$ and of hydrogen on the $\mathrm{Si}(111)$ surface also yields results in good agreement with first-principles calculations and experiments.
\end{abstract}

\section{INTRODUCTION}

Hydrogenated silicon systems have been intensely studied for their importance in device technology and also for their array of interesting physical phenomena. Hydrogen is commonly used for passivating danglingbond defects in hydrogenated amorphous silicon systems ${ }^{1}$ as well as in hydrofluoric-acid-etched crystalline silicon surfaces. ${ }^{2}$ It has been found in experiments that the hydrogen causes changes in the electrical and optical properties, as well as the surface structures. ${ }^{3}$ Nevertheless, the physical mechanism underlying these phenomena is still far from being completely understood. Due to the complexity of the systems, theoretical studies have been a great challenge.

First-principles techniques such as local-densityfunctional formalism for the problem have been developed and have produced some significant results. ${ }^{4-7}$ However, the computational cost is prohibitive for large systems. Even though recent breakthroughs such as the Car-Parrinello method ${ }^{8}$ have made molecular dynamics possible within the local-density-functional regime, the simulation is still limited to a small number of atoms $(\sim$ 130 atoms for the H-Si system ${ }^{7}$ ) and to short times (a few picoseconds).

On the other hand, there are a few empirical classical interatomic potentials for hydrogen-silicon systems that have been proposed. ${ }^{9,10}$ These potentials have been used to build a computer model of amorphous silicon hydrides. ${ }^{9,11}$ Computational cost is not a problem here, but the reliability of the classical potentials in treating the dynamics of hydrogen-silicon systems is still an open question.

Several years ago, Allan and Mele extended the empirical tight-binding scheme, which has been quite successful for pure silicon systems, ${ }^{12}$ to the study of hydrogenated Si surfaces. ${ }^{13}$ The advantage of the tightbinding potential over the classical empirical potentials is that the quantum-mechanical bonding nature of the system is taken into account through the underlying electronic structure rather than through ad hoc $N$-body potentials. ${ }^{9-11,14-17}$ The tight-binding scheme is computationally efficient since it uses a minimal basis for the electronic calculations and the Hamiltonian matrix elements are parametrized. With the Chadi tight-binding model $^{18}$ for silicon-silicon interaction and a hydrogensilicon interaction derived from the silane molecule, ${ }^{13}$ Allan and Mele have produced some reasonable results for the $T=0$ properties of the hydrogenated silicon surfaces. However, the tight-binding model used by Allan and Mele has some drawbacks when considered for use in molecular-dynamics simulations. Firstly, the symmetric and antisymmetric bending modes of silane $\left(\mathrm{SiH}_{4}\right)$ predicted by their model are about $30 \%$ smaller than those of the experimental data. Secondly, the model is constructed in the harmonic regime and is not adequate for use in molecular-dynamics simulations that study the properties of the system at high temperatures, where the anharmonic effects on the dynamics of hydrogens are very important. Furthermore, because of the large amplitude of the zero-point motion of the hydrogen atom, anharmonic effects are not negligible even at very low temperatures.

In this paper, we propose an improved tight-binding model designed for use in molecular-dynamics simulations of the hydrogen-silicon systems. The hydrogensilicon interaction is also derived from the silane $\left(\mathrm{SiH}_{4}\right)$ molecule proceeding in a way similar to that of Allan and Mele. However, our model includes anharmonic interactions and also reproduces accurately the vibration frequencies of the silane molecule. Further tests on disilane molecules and $\mathrm{H}$ on the $\mathrm{Si}(111)$ surface suggest that the model has very good transferability.

\section{TIGHT-BINDING MODEL FOR HYDROGEN-SILICON INTERACTION}

In an empirical tight-binding (ETB) scheme, ${ }^{18^{-24}}$ the total energy is written as

$$
E_{\mathrm{tot}}=E_{\mathrm{bs}}+U
$$

where

$$
E_{\mathrm{bs}}=\sum_{n, \mathbf{k}} \varepsilon_{n, \mathbf{k}}
$$


is the band-structure energy consisting of a summation of occupied one-electron eigenenergies calculated by the Slater-Koster-type empirical tight-binding method ${ }^{25}$ and $U$ is a short-ranged repulsive potential energy. $U$ may be determined by subtracting $E_{\mathrm{bs}}$ from $E_{\text {tot }}$ for a simple special case where data are known either from firstprinciples total-energy calculations or from experiments. The success of this ETB model in studying the anharmonic properties of Si (Ref. 19) could be attributed to the incorporation of detailed accurate total-energy information.

In a $\mathrm{Si}-\mathrm{H}$ system, one needs to consider interaction parameters for three types of pairs, $\mathrm{Si}-\mathrm{Si}, \mathrm{Si}-\mathrm{H}$, and $\mathrm{H}-$ $\mathrm{H}$. For Si-Si pairs, we use the nearest-neighbor tightbinding potential model previously developed ${ }^{19}$ in which the tight-binding parameters are taken from Chadi's work ${ }^{18}$ and the repulsive potential is derived from the first-principles total-energy calculation results of Yin and Cohen. ${ }^{26}$ The tight-binding parameters are given as follows:

$$
\begin{aligned}
& E_{s}=-13.08 \mathrm{eV}, E_{p}=-6.63 \mathrm{eV}, \\
& V_{s s \sigma}(r)=-1.938\left(r_{0} / r\right)^{2} \mathrm{eV}, \\
& V_{s p \sigma}(r)=1.745\left(r_{0} / r\right)^{2} \mathrm{eV} \\
& V_{p p \sigma}(r)=3.05\left(r_{0} / r\right)^{2} \mathrm{eV}, \\
& V_{p p \pi}(r)=-1.075\left(r_{0} / r\right)^{2} \mathrm{eV},
\end{aligned}
$$

where $r$ is the interatomic distance and $r_{0}=2.36 \AA$ is the nearest-neighbor distance of silicon in diamond structure. Molecular-dynamics studies using this potential have produced a quantitative description of the temperaturedependent frequency shifts and phonon linewidths of the phonons in crystalline $\mathrm{Si}^{19}$

For Si-H pairs the tight-binding parameters are fitted to the occupied eigenvalues and the symmetric bending mode vibration frequency of the silane $\left(\mathrm{SiH}_{4}\right)$ molecule, proceeding in a way similar to that of Allan and Mele. ${ }^{13}$ We obtained

$$
\begin{aligned}
& E_{s}=-8.0 \mathrm{eV} \\
& V_{s s \sigma}=-3.63 \mathrm{eV}, \quad V_{s p \sigma}=4.63 \mathrm{eV} .
\end{aligned}
$$

The bond-length dependence of the hopping parameters is also assumed to follow the $1 / r^{2}$ scaling law. ${ }^{20}$ Unlike the previous work of Allan and Mele, ${ }^{13}$ we do not require the model to reproduce the eigenvalues of the antibonding states in silane. We believe that the eigenvalues of the empty states are not well described with a minimal basis set for $\mathrm{Si}$. This is consistent with the fact that the Chadi model gives a poor description of the conduction bands in crystalline Si. Thus we obtain results which are higher than the experimental values. We think further improvement of the basis set by including extra orbitals for $\mathrm{Si}$ will bring the empty states lower towards the experimental value. However, since the antibonding states are far away from the Fermi level, this will not strongly affect the energetics of the system. We find that $V_{s p \sigma}$ is now much larger than Allan and Mele's, providing more realistic bond-bending forces. This improvement will be important for the bond-bending modes where only the tight-binding term is contributing to the restoring force.

We use the universal binding curve ${ }^{27}$ to parametrize the total energy per bond $E_{\text {tot }}$ of the silane $\left(\mathrm{SiH}_{4}\right)$ molecule as a function of $\mathrm{Si}-\mathrm{H}$ bond length.

$E_{\text {tot }}=-E_{0}(1+x) e^{-x}, x=\left(r-d_{0}\right) / l_{s}$, where $E_{0}=3.355$ $\mathrm{eV}$ and $d_{0}=1.48 \AA$ are the experimental values of bond energy ${ }^{28}$ and equilibrium bond length of silane, and the scaling length $l_{s}=0.4264 \AA$ is fitted to the vibration frequency (271.4 meV) of the symmetric stretching mode of silane with the procedure that will be described in the next paragraph. The repulsive potential between the hydrogen-silicon pair is then determined by subtracting the bond-length dependence of $E_{\mathrm{bs}}$ of silane from the above universal binding curve. The significance of this formulation is that anharmonic information is included in the two-body repulsive potential as well as the tightbinding term. This is essential for use in a moleculardynamics simulation. Since Allan and Mele make the expansion of the two-body repulsive potential only up to the second order, their model is not adequate for a molecular-dynamics simulation.

Due to the light mass of the hydrogen atom, we find

TABLE I. Comparison of calculated $\mathrm{SiH}_{4}$ eigenvalues (in $\mathrm{eV}$ ) and bending mode frequencies (in $\mathrm{meV}$ ) with experiment (Ref. 31) and other theoretical calculations (Ref. 13).

\begin{tabular}{lccc}
\hline Mode & Experiment & Allan and Mele (Ref. 13) & Present work \\
\hline$a_{1}^{-}$ & -4.10 & -5.17 & -2.84 \\
$t_{2}^{-}$ & -5.40 & -4.95 & -1.93 \\
$t_{2}^{+}$ & -12.70 & -12.59 & -12.71 \\
$a_{1}^{+}$ & -18.20 & -18.82 & -18.23 \\
Symmetric & 121 & 71.7 & 122 \\
Bending & 113 & 72.6 & 103 \\
Antisymmetric & & & \\
Bending & & & \\
\hline \hline
\end{tabular}


TABLE II. Comparison of calculated $\mathrm{Si}_{2} \mathrm{H}_{6}$ eigenvalues (in eV) and the frozen phonon calculation result of hydrogen vibration frequencies in disilane molecule (in meV). With the consideration of the zero-point energy, the stretching mode frequency should decrease by about $5 \mathrm{meV}$.

\begin{tabular}{lrrr}
\hline \hline & Experiment & Allan and Mele (Ref. 13) & $\begin{array}{r}\text { Present } \\
\text { calculation }\end{array}$ \\
\hline$a_{g}$ & -10.7 & -10.93 & -11.07 \\
$e_{g}$ & -12.1 & -12.38 & -12.28 \\
$e_{u}$ & -13.3 & -12.85 & -13.23 \\
$a_{u}$ & -17.3 & -17.27 & -16.65 \\
$a_{g}$ & & -19.18 & -18.71 \\
& & & \\
Stretching & 248 & & 276 \\
Wagging & 78 & & 75 \\
Twist & 62 & & 55 \\
\hline \hline
\end{tabular}

that it is important to include the zero-point motion of the hydrogen atom in our molecular-dynamics simulation to obtain an accurate description of the experimental phonon frequency shifts and phonon linewidths for the hydrogen vibration. Because of the large amplitude of the hydrogen zero-point motion, we have found that the frequency of the stretching mode frequency of silane obtained from the molecular-dynamics simulation at low temperature is shifted to lower frequency by about $5 \mathrm{meV}$ compared to that obtained by frozen-phonon calculations performed with small displacements. Since we want to use our model in a molecular-dynamics simulation, we choose to include the large vibration amplitude of hydrogen at the earliest stage in the fitting of the stretching mode of hydrogen in silane for consistency. For fitting the parameter $l_{s}$, simulation is performed for a hydrogen atom moving in a potential well given by the universal binding curve, with an initial distortion amplitude estimated from the zero-point energy of hydrogen in the given well. The parameter $l_{s}$ is adjusted such that the frequency of the hydrogen obtained from the moleculardynamics simulation is equal to $271.4 \mathrm{meV}$. For comparison, the frozen phonon calculation with a small displacement would give $276.5 \mathrm{meV}$, which would be $5 \mathrm{meV}$ higher than the experimental value.

In this paper the hydrogen-hydrogen interaction is neglected. This approximation is adequate for systems where the $\mathrm{H}-\mathrm{H}$ interatomic distances are large [for example, the monohydride phase on $\mathrm{Si}(111)$ where the $\mathrm{H}-\mathrm{H}$ distance is $3.84 \AA$ ]. H-H interaction may be needed for systems where the hydrogen atoms are much closer to each other, for example in cases where dihydride and trihydride phases exist.

\section{RESULTS AND DISCUSSION}

Results of the energy levels and vibrational frequencies of the silane $\left(\mathrm{SiH}_{4}\right)$ molecule calculated by the above tight-binding model are listed in Table I in comparison with experimental data and the previous tight-binding calculation of Allan and Mele. ${ }^{13}$ The eigenvalues of the bonding states $\left(a_{1}^{+}\right.$and $\left.t_{2}^{+}\right)$agree very well with the experiment, while the eigenvalues of the antibonding states $\left(t_{2}^{-}\right.$and $\left.a_{1}^{-}\right)$are higher than the experimental values. We attribute the poor results for the antibonding states to the use of only $s p^{3}$ orbitals for silicon atoms. If one includes also $d$ orbitals to represent the electronic states of silicon atoms, the antibonding energy levels would be lower than the present values. Judging from past experience with silicon and carbon systems, an accurate description of the bonding states should be sufficient for studying the structural properties of the system. The bending mode frequencies are decided solely by the TB parameters and not related to the two-body potential. The symmetric bending mode is in excellent agreement with experiment, while the asymmetric bending mode is about $10 \%$ less than the experimental value. Results for the disilane $\left(\mathrm{Si}_{2} \mathrm{H}_{6}\right)$ molecule are summarized in Table II with the experimental vibration frequencies. ${ }^{29}$

Having established the model, we proceed to calculate the electronic properties, surface relaxations, and the phonon vibration frequencies of the hydrogenated $\mathrm{Si}(111)$ surface. We have chosen a slab in the (111) direction which has $12 \mathrm{Si}$ layers in the case of the monohydride surface. Two hydrogen layers are added on the top and bottom of the slab (Fig. 1). We have used 120 and 55 $\mathbf{k}$ points, respectively, in the irreducible part of the first Brillouin zone to calculate the surface electronic density of states and band-structure energy $E_{\mathrm{bs}}$. The convergence of the above $\mathbf{k}$-point samplings has been verified

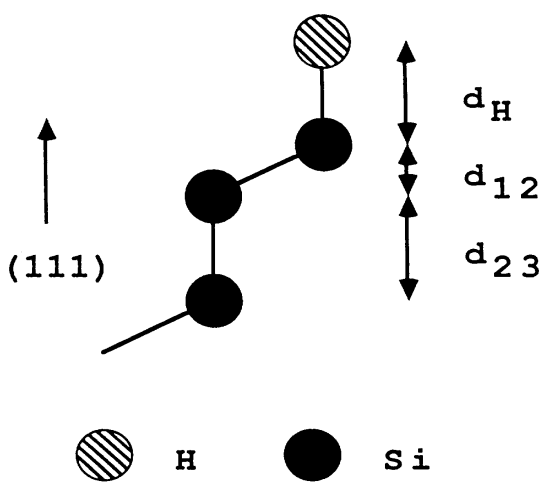

FIG. 1. Surface geometry of the monohydride Si(111) surface. 
TABLE III. Surface relaxations of the monohydride $\mathrm{Si}(111)$ surface compared with the first-principles calculation result. "Ideal" corresponds to the case without any surface relaxations. All lengths are in angstroms. Interplanar spacings $d_{\mathrm{H}}, d_{12}$, and $d_{23}$ are defined in Fig. 1. The asterisk denotes the sum of the covalent radii of $\mathrm{Si}$ and $\mathrm{H}$. We take this to be the bond length between $\mathrm{Si}$ and $\mathrm{H}$ in the silane $\left(\mathrm{SiH}_{4}\right)$ molecule.

\begin{tabular}{llll}
\hline \hline & $\begin{array}{l}\text { Present } \\
\text { calculation }\end{array}$ & $\begin{array}{l}\text { First-principles } \\
\text { calculation (Ref. 6) }\end{array}$ & Ideal \\
\hline$d_{\mathrm{H}}$ & 1.49 & 1.54 & $1.48^{*}$ \\
$d_{12}$ & 0.81 & 0.72 & 0.78 \\
$d_{23}$ & 2.35 & 2.32 & 2.35 \\
$d_{12}$, clean & 0.79 & 0.57 & 0.78 \\
$d_{23}$, clean & 2.39 & & 2.35 \\
\hline \hline
\end{tabular}

to be enough for both the clean $\mathrm{Si}(111)$ surface and the monohydride surface.

Shown in Fig. 2 is the local electronic density of states (LDOS) for the Si(111) monohydride surface. The arrows indicate the prominent hydrogen-related peaks. The LDOS's have a characteristic three-peak feature below the Fermi energy, with the peak at the lowest, medium, and highest energy having $s$-like, $s p$-like, and $p$-like characters, respectively. It compares quite well with the re-

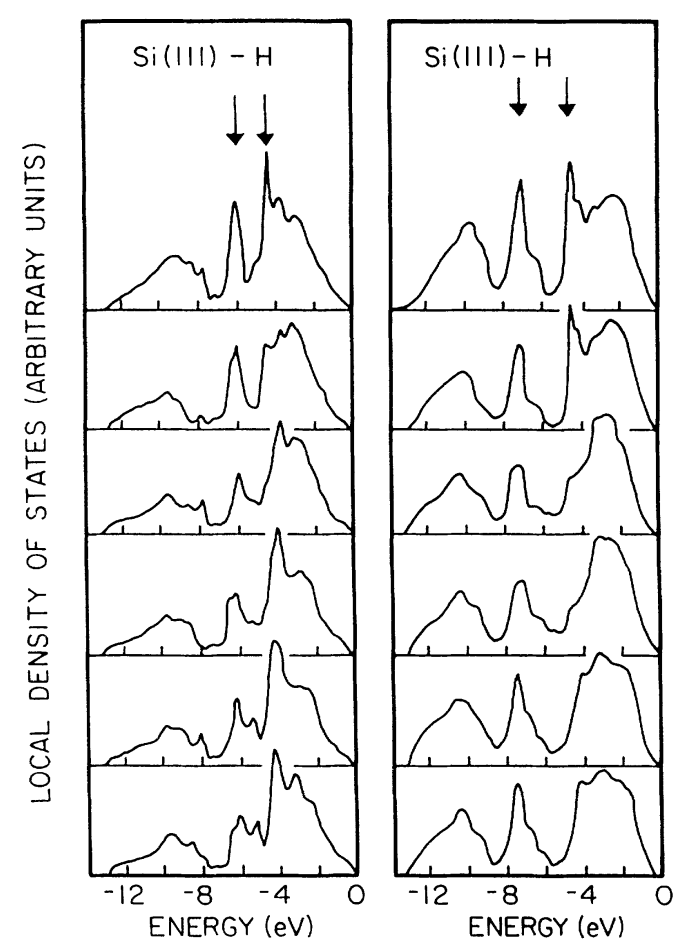

FIG. 2. Local electronic density of states for the monohydride $\operatorname{Si}(111)$ surface from the present ETB calculation (left) compared with the first-principles result (Ref. 30) (right). The top-row figure corresponds to the hydrogen layer and the outermost Si layer. The second-row figure corresponds to the second Si layer, and so on.
TABLE IV. Hydrogen vibration frequencies on the monohydride $\mathrm{Si}(111)$ surface in meV. Our result for the stretching mode includes the zero-point motion of hydrogen. The first-principles calculation is a small amplitude calculation in which case the frequency would decrease upon consideration of the zero-point motion of hydrogen.

\begin{tabular}{lrrr}
\hline \hline & $\begin{array}{c}\text { Present } \\
\text { calculation }\end{array}$ & $\begin{array}{r}\text { First-principles } \\
\text { calculation (Ref. 6) }\end{array}$ & Experiment \\
\hline Stretching & 270 & 245 & 257 \\
Wagging & 74 & 71 & 77 \\
\hline \hline
\end{tabular}

sults from more-accurate self-consistent calculations. ${ }^{30}$

Results of surface relaxation of the monohydride Si(111) surface are presented and compared with firstprinciples calculation results in Table III. In our calculations, the hydrogen layer and two outermost Si layers are allowed to relax in the (111) direction only. For the monohydride $\mathrm{Si}(111)$ surface, the present tight-binding model predicts slightly outward relaxation of the top layer of silicon, in contrast with the first-principles calculation result, which shows slight contraction. The equilibrium bond length between hydrogen and silicon of the monohydride phase is $1.49 \AA$ from the tight-binding calculation, which is smaller than the first-principles result of $1.54 \AA .6$

Finally, the vibration frequencies of hydrogen on the monohydride $\mathrm{Si}(111)$ surface are presented in Table IV. The frequencies of the stretching and wagging modes predicted by the present tight-binding model are in excellent agreement with experimental data and first-principles calculation results. For the trihydride $\mathrm{Si}(111)$ surface, the symmetric bending mode frequency $93 \mathrm{meV}$ is about $15 \%$ lower than the experimental value $108 \mathrm{meV}$. Since in the trihydride phase the distance between the hydrogen pair is smaller (about $2.2 \AA$ ), the neglect of the $\mathrm{H}$-H interactions may cause the underestimation for the bending mode frequencies.

To summarize, we have established an empirical tightbinding model for the study of the silicon hydrogen system. Our model gives good results for the hydrogenated $\mathrm{Si}(111)$ surface. In particular, the vibrational frequencies of hydrogen are reproduced in a satisfactory way. The simplicity of the model makes it applicable for study in a problem where a large system has to be considered, such as surface problems and amorphous silicon.

\section{ACKNOWLEDGMENTS}

Ames Laboratory is operated by Iowa State University for the U.S. Department of Energy under Contract No. W-7405-ENG-82. This research is supported by the director for Energy Research, Division of Material Sciences, U.S. Department of Energy, and by the National Science Foundation under Grant No. DMR-8819379. One of us (Y.H.L.) would like to thank Ames Laboratory and Microelectronics Research Center for their hospitality and support, and Korea Science and Engineering Foundation and Ministry of Education of Korea for their support. 
*Permanent address: Department of Physics, Jeonbug National University, Jeonju, Jeonbug 560-756, Korea.

${ }^{1}$ For example, see the review article by S. J. Pearton, J. W. Corbett, and T. S. Shi, Appl. Phys. A 43, 153 (1987).

${ }^{2}$ H. Ubara, T. Imura, and A. Hiraki, Solid State Commun. 50, 673 (1984); M. Grundner and H. Jacob, Appl. Phys. A 39, 73 (1986); E. Yablonovitch, D. L. Allara, C. C. Chang, T. Gmitter, and T. B. Bright, Phys. Rev. Lett. 57, 249 (1986). G. S. Higashi, Y. J. Chabal, G. W. Trucks, and K. Raghavachari, Appl. Phys. Lett. 56, 656 (1990).

${ }^{3}$ For example, see the review article by D. Haneman, Rep. Prog. Phys. 50, 1045 (1987), and the references therein.

${ }^{4}$ K. J. Chang and D. J. Chadi, Phys. Rev. Lett. 60, 1422 (1980); Phys. Rev. B 40, 11644 (1989).

${ }^{5}$ C. G. Van de Walle, Y. Bar-Yam, and S. T. Pantelides, Phys. Rev. Lett. 60, 2761 (1988); C. G. Van de Walle, P. J. H. Denteneer, Y. Bar-Yam, and S. T. Pantelides, Phys. Rev. B 39, 10791 (1989).

${ }^{6}$ E. Kaxiras and J. D. Joannopoulos, Phys. Rev. B 37, 8842 (1988).

${ }^{7}$ F. Buda, G. L. Chiarotti, R. Car, and M. Parrinello, Phys. Rev. Lett. 63, 294 (1989).

${ }^{8}$ R. Car and M. Parrinello, Phys. Rev. Lett. 55, 2471 (1985).

${ }^{9}$ L. Guttman, Phys. Rev. B 23, 1866 (1981); L. Guttman and C. Y. Fong, ibid. 26, 6756 (1982).

${ }^{10}$ A. Selmani, D. R. Salahub, and A. Yelon, Surf. Sci. 202, 269 (1988).

${ }^{11}$ L. J. Lewis, N. Mousseau, and F. Drolet, in Atomic-Scale Calculations in Materials Science, Materials Research Society Symposium Proceedings Vol. 144, edited by J. Tersoff, D. Vanderbilt, and V. Vitek (MRS, Pittsburgh, 1989); N. Mousseau and L. J. Lewis, Phys. Rev. B 41, 3702 (1990).

${ }^{12}$ D. C. Allan and E. J. Mele, Phys. Rev. Lett. 53, 826 (1984); Phys. Rev. B 35, 5533 (1987).
${ }^{13}$ D. C. Allan and E. J. Mele, Phys. Rev. B 31, 5565 (1985).

${ }^{14}$ P. N. Keating, Phys. Rev. 145, 637 (1966).

${ }^{15}$ F. H. Stillinger and T. A. Weber, Phys. Rev. B 31, 5262 (1985).

${ }^{16}$ R. Biswas and D. R. Hamann, Phys Rev. B 36, 6434 (1987).

${ }^{17}$ J. Tersoff, Phys. Rev. Lett. 56, 632 (1986).

${ }^{18}$ D. J. Chadi, Phys. Rev. Lett. 41, 1062 (1978); Phys. Rev. B 29, 785 (1984); Phys. Rev. Lett. 59, 1691 (1988).

${ }^{19}$ C. Z. Wang, C. T. Chan, and K. M. Ho, Phys. Rev. B 39, 8586 (1989); 40, 3390 (1989); 42, 11276 (1990); C. Xu, C. Z. Wang, C. T. Chan, and K. M. Ho, ibid. 43, 5024 (1991).

${ }^{20}$ W. A. Harrison, Electronic Structure and the Properties of Solids (Freeman, San Francisco, 1980).

${ }^{21}$ D. Tomanék and M. A. Schlüter, Phys. Rev. Lett. 56, 1055 (1986); Phys. Rev. B 36, 1208 (1987).

${ }^{22}$ D. G. Allan and E. J. Mele, Phys. Rev. Lett. 53, 826 (1984); O. L. Alerhand, D. G. Allan, and E. J. Mele, ibid. 55, 2700 (1985).

${ }^{23}$ F. S. Khan and J. Q. Broughton, Phys. Rev. B 39, 3688 (1989).

${ }^{24}$ M. Menon and R. E. Allen, Phys. Rev. B 33, 7099 (1986); 38, 6196 (1988).

${ }^{25}$ J. C. Slater and G. F. Koster, Phys. Rev. 94, 1498 (1954).

${ }^{26}$ M. T. Yin and M. L. Cohen, Phys. Rev. B 26, 5668 (1982).

${ }^{27}$ J. H. Rose, J.Ferrante, and J. R. Smith, Phys. Rev. Lett. 47, 675 (1981).

${ }^{28}$ S. R. Gunn and L. G. Green, J. Phys. Chem. 65, 779 (1961); D. S. Horowitz and W. A. Godd ard III, J. Mol. Struct. 163, 207 (1988).

${ }^{29}$ G. Lucovsky, R. J. Nemanich, and J. C. Knights, Phys. Rev. B 19, 2064 (1979).

${ }^{30}$ K. M. Ho and M. L. Cohen, Phys. Rev. B 15, 3888 (1977).

${ }^{31}$ See the review article by M. Cardona, Phys. Status Solidi B 118, 463 (1983). 